\title{
Widening the Neuroimaging Features of Adenosine Deaminase 2 Deficiency
}

\author{
(D) A.F. Geraldo, (D) R. Caorsi, (DD. Tortora, (D). Gandolfo, (D). Ammendola, (D). Alessio, (D) G. Conti, (D)A. Insalaco, (D). Pastore,
} (D) S. Martino, (D). Ceccherini, (D) S. Signa, (D) M. Gattorno, (D) A. Rossi, and (D) M. Severino

\begin{abstract}
SUMMARY: Adenosine deaminase 2 deficiency (OMIM \#615688) is an autosomal recessive disorder characterized by a wide clinical spectrum, including small- and medium-sized vessel vasculopathies, but data focusing on the associated neuroimaging features are still scarce in the literature. Here, we describe the clinical neuroimaging features of 12 patients with genetically proven adenosine deaminase 2 deficiency (6 males; median age at disease onset, 1.3 years; median age at genetic diagnosis, 15.5 years). Our findings expand the neuroimaging phenotype of this condition demonstrating, in addition to multiple, recurrent brain lacunar ischemic and/or hemorrhagic strokes, spinal infarcts, and intracranial aneurysms, also cerebral microbleeds and a peculiar, likely inflammatory, perivascular tissue in the basal and peripontine cisterns. Together with early clinical onset, positive family history, inflammatory flares and systemic abnormalities, these findings should raise the suspicion of adenosine deaminase 2 deficiency, thus prompting genetic evaluation and institution of tumor necrosis factor inhibitors, with a potential great impact on neurologic outcome.
\end{abstract}

ABBREVIATIONS: DADA2 = adenosine deaminase 2 deficiency; TNF = tumor necrosis factor; PAN = polyarteritis nodosa; PRES = posterior reversible encephalopathy syndrome

A denosine deaminase 2 deficiency (DADA2) is an autosomal recessive disorder (OMIM \#615688) usually presenting in the pediatric age group. ${ }^{1,2}$ It is characterized by a wide clinical spectrum, including systemic autoinflammation, polyarteritis nodosa (PAN)-like vasculopathy, noninflammatory medium-

Received August 22, 2020; accepted after revision November 23.

From the Neuroradiology Unit (A.F.G., D.T., R.A., A.R., M.S.), Center for Autoinflammatory Diseases and Immunodeficiencies (R.C., S.S., M.G.), and Interventional Unit (C.G.), IRCCS Istituto Giannina Gaslini, Genoa, Italy; Diagnostic Neuroradiology Unit, Imaging Department (A.F.G.), Centro Hospitalar Vila Nova de Gaia/Espinho, Vila Nova de Gaia, Portugal; Department of Translational Medical Sciences (M.A.), Federico II University of Naples, Naples, Italy; Pediatric

Nephrology and Rheumatology Unit (G.C.), AOU G Martino, Messina, Italy; Division of Rheumatology (A.I.), IRCCS Ospedale Pediatrico Bambino Gesù, Roma, Italy; Department of Pediatrics (S.P.), Institute for Maternal and Child Health - IRCCS "Burlo Garofolo", Trieste, Italy; Division of Pediatric Immunology and Rheumatology, Department of Public Health and Pediatrics (S.M.), Regina Margherita Children Hospital, University of Turin, Turin, Italy; UOSD Genetics and Genomics of Rare Diseases (I.C.), IRCCS Istituto Giannina Gaslini, Genoa, Italy; and Department of Health Sciences (DISSAL) (A.R.), University of Genoa, Genoa, Italy. A.F.Geraldo and R.Caorsi contributed equally to this work.

This work was supported by funds from Ricerca Corrente Disordini Neurologici e Muscolari (Linea 5) of the Italian Ministry of Health and by the Compagnia di San Paolo (Grant ROL 20573).

Paper previously presented as a poster at: 8th International Congress of Familial Mediterranean Fever and Systemic Autoinflammatory Diseases, September 30 to October 3, 2015; Dresden, Germany.

Ethics approval: All procedures performed in studies involving human participants were in accordance with ethical standards of the institutional and/or national research committee and with the 1964 Helsinki declaration and its later amendments or comparable ethical standards.

Informed consent was waived because of the retrospective nature of the study. vessel arteriopathy, and pure hematologic disorders without vasculopathy. ${ }^{1-5}$

The ADA2 gene (previously known as CECR1) is located at 22q11.1 and encodes for ADA2, a dimeric extracellular enzyme primarily secreted by cells of the myeloid lineage, converting adenosine in inosine. ${ }^{1,2}$ In addition to its catalytic function, ADA2 presents antiinflammatory and immunomodulatory properties and has a role in maintenance of vascular integrity. ${ }^{2}$

CNS involvement caused by small- and medium-sized vessel vasculopathy is a well-recognized complication, being present in nearly $50 \%$ of cases, ${ }^{6}$ mostly without anomalies detectable on angiographic studies. ${ }^{2,6-9}$ Neurologic events usually occur early in the course of the disease, at a very young age, ${ }^{1,2,6,10}$ frequently associated with inflammatory flares. ${ }^{2,10}$ Affected patients may present with ischemic strokes, distributed in the territory of perforator arteries, or intracranial hemorrhages, often recurrent, leading to clinical manifestations varying from minimal neurologic deficits to fatal outcome. ${ }^{1,2,6,8,10-12}$ Clinically silent brain lesions and TIAs with negative MR imaging findings may also occur, ${ }^{7}$ as well as peripheral neuropathy, acute sensorineural

Please address correspondence to Domenico Tortora, MD, PhD, Neuroradiology Unit, IRCCS Istituto Giannina Gaslini, Via Gaslini 5, Genova 16148, Italia; e-mail: domenicotortora@gaslini.org; @TortoraDomenico; @DrGeraldoFilipa

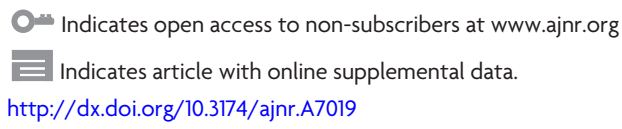

AJNR Am J Neuroradiol 42:975-79 May 2021 www.ajnr.org 
hearing loss, optic neuritis, and other ophthalmologic abnormalities. $^{1,2,6-8,10,11}$

Knowledge of DADA2-related neuroimaging patterns is of particular importance because CNS manifestations are considered a marker of severity and may be the presenting form of DADA2. ${ }^{4,9,10}$ Moreover, this may prompt early treatment with tumor necrosis factor (TNF) inhibitors, leading to complete remission and preventing further neurologic events. ${ }^{13}$ However, neuroimaging features associated with this condition are still incompletely elucidated. Indeed, to date, only 1 case series has been published, including neuroimaging data of 12 patients with DADA2, with limited information regarding angiographic features and radiologic follow-up. ${ }^{14}$

Recently, we encountered novel neuroimaging findings in patients with DADA2 that could be part of the spectrum of anomalies associated with this syndrome. Therefore, we aimed to provide a detailed description of CNS lesions and their long-term evolution in a series of 12 patients with genetically confirmed diagnosis of DADA2, as well as of the corresponding clinical, laboratory, and genetic data.

\section{CASE SERIES}

Twelve patients with DADA2 from 9 different families observed between 2014 and 2019 in 6 Italian hospitals were included in this case series. Institutional review board approval was obtained, waiving parental written informed consent. Demographic information, medical history, laboratory and histopathologic results, and genotypes were retrieved from electronic medical records. Clinical data are summarized in the Table and the Online Supplemental Data.

Details on neuroimaging studies and protocols are presented in the Online Supplemental Data. Images were analyzed by 2 pediatric neuroradiologists in consensus (M.S. and A.F.G., with 10 and 5 years' experience). Neuroimaging features are reported in the Online Supplemental Data.

At first brain MR imaging, 9/12 patients (75\%) presented small focal areas of T2/FLAIR signal abnormality with or without restricted diffusion compatible with lacunar infarcts caused by small-vessel occlusions (Online Supplemental Data). Of these, 7 (77.8\%) had multiple ischemic lesions, and 1 concomitantly presented with a large chronic hemorrhagic lesion in the left temporal lobe. Both anterior and posterior circulations were involved in $4 / 9$ cases, and either the anterior or the posterior circulation was involved in 3/9 and 2/9 patients, respectively. The nucleocapsular region was the more severely affected region (11 lesions, 5 patients) followed by the midbrain ( 9 lesions, 5 patients) and the thalamus (6 lesions, 2 patients). One patient exhibited isolated intracranial hemorrhagic lesions, and 2 presented with small foci of susceptibility effect in the parenchyma and another 1 in the interpeduncular cistern (Fig 1). Two additional patients showed clinicoradiologic features compatible with posterior reversible encephalopathy syndrome (PRES) (Online Supplemental Data).

Follow-up MR imaging detected new cerebral infarcts in 5/10 patients (50\%), and 1 of them also presented an anterior spinal artery infarct at the cervical level (Online Supplemental Data). All events occurred before institution of anti-TNF or thalidomide except 1 identified shortly after introduction of anti-TNF and another during temporary discontinuation of thalidomide. In
Clinical data of patients with DADA2

\begin{tabular}{|c|c|}
\hline Features & Results \\
\hline Males & $6 / 12$ \\
\hline Positive family history & $7 / 11^{\mathrm{a}}$ \\
\hline \multicolumn{2}{|l|}{ Median age (years, range) at: } \\
\hline Disease onset & $1.3(0.25-7)$ \\
\hline Genetic diagnosis & $15.5(5-35)$ \\
\hline Neurologic onset & $5.5(0.5-12)$ \\
\hline First stroke $(n=10)$ & $6.5(1.4-12)$ \\
\hline \multicolumn{2}{|l|}{ Symptoms at presentation } \\
\hline Fever & $5 / 12$ \\
\hline Skin manifestations & $4 / 12$ \\
\hline Neurologic problems & $4 / 12$ \\
\hline \multicolumn{2}{|l|}{ Disease course } \\
\hline Chronic & $11 / 12$ \\
\hline Recurrent & $1 / 12$ \\
\hline \multicolumn{2}{|l|}{ Manifestations } \\
\hline Neurologic disorders & $12 / 12$ \\
\hline Hypertension & $12 / 12$ \\
\hline Elevation of acute phase reactants & $12 / 12$ \\
\hline Inflammatory skin lesions & $12 / 12$ \\
\hline Musculoskeletal disorders & $5 / 12$ \\
\hline Gastrointestinal abnormalities & $5 / 12$ \\
\hline Hypogammaglobulinemia & $5 / 12^{b}$ \\
\hline Cardiac abnormalities & $2 / 12$ \\
\hline \multicolumn{2}{|l|}{ Genetics ${ }^{c}$} \\
\hline $\begin{array}{l}\text { Homozygous or compound hemizygous } \\
\text { ADA2 variants }\end{array}$ & $11 / 12$ \\
\hline Homozygous tandem 22q.11.1 duplication & $1 / 12$ \\
\hline \multicolumn{2}{|l|}{ Skin/bowel biopsy } \\
\hline Polyarteritis nodosa & $5 / 7$ \\
\hline Leukocytoclastic vasculitis & $2 / 7$ \\
\hline \multicolumn{2}{|l|}{ Treatment $^{\mathrm{d}}$} \\
\hline Anti-TNF & $9 / 12$ \\
\hline Thalidomide & $3 / 12$ \\
\hline
\end{tabular}

addition, $3 / 9$ patients (33\%) with ischemic strokes developed an abnormal contrast-enhancing soft tissue in the interpeduncular cistern, encasing the midbrain perforating arteries, with associated areas of eccentric arterial wall enhancement and thickening, that completely subsided after anti-TNF treatment (Fig 2, Online Supplemental Data). In addition, 1 patient with PRES presented multiple extraaxial foci of enhancement in the left crural cistern and inner auditory canal (Online Supplemental Data). Arterial spinlabeling studies revealed normal, symmetric cerebral perfusion in $7 / 7$ cases except in the regions corresponding to the infarcts.

Qualitative assessment of the CSF spaces revealed mild diffuse enlargement of the ventricular system, the cortical cerebral sulci, or both in 5/12 (42\%) patients at first brain MR imaging. None of the 10 patients with follow-up studies presented signs of progressive atrophy.

Intracranial arterial abnormalities were noted before treatment in 2/12 patients, including small-sized intracranial aneurysms in the superior cerebellar artery and anterior communicating artery, spontaneously thrombosed at follow-up (Fig 3), and transient stenosis of the left posterior communicating artery associated with contrast-enhancing perivascular tissue. Renal Doppler/abdominal CTA and echocardiography were performed in 11/12 and 7/12 patients, respectively, including the patient with intracranial aneurysms, but no visceral aneurysms were found. 


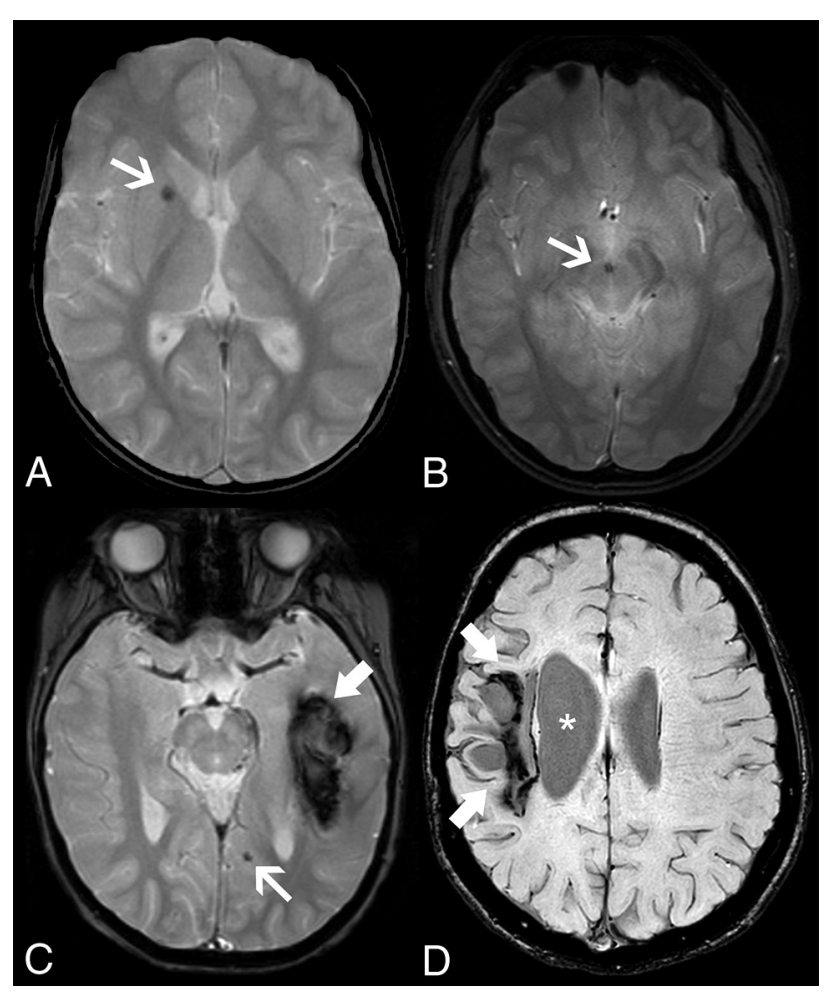

FIG 1. Intracranial hemorrhagic manifestations in adenosine deaminase 2 deficiency. Axial gradient-echo T2-weighted images of patient 4 at 5.8 years of age $(A)$ and patient 11 at 18.3 years of age $(B)$ demonstrate microbleeds in the right lenticular nucleus (arrow) and in the interpeduncular cistern or vessel wall (arrow), respectively. Axial gradient-echo T2-weighted image $(C)$ of patient 1 at 6.1 years of age reveals an acute left temporal hematoma (thick arrow) and a left paramedian occipital microbleed (arrow). None of the patients presented with head CT calcifications in the corresponding locations of the microbleeds (not shown). Axial SWI of patient $5(D)$ at 16.5 years of age depicts a right frontoparietal hemorrhagic chronic lesion (thick arrows) causing ex vacuo dilation of the lateral ventricle (asterisks).

\section{DISCUSSION}

In this series, we identified a wide spectrum of neuroimaging abnormalities associated with DADA2, including 2 novel imaging patterns. All patients presented a vascular phenotype, as expected in most patients harboring missense mutations, ${ }^{5}$ but none had hematologic disorders besides hypogammaglobulinemia. Neurologic involvement at presentation was common (33\% of cases). As previously shown, ${ }^{2,14}$ small, multiple ischemic infarcts in the nucleocapsular and mesencephalic or thalamic regions were the most frequent MR imaging abnormality, being present in $75 \%$ of our patients and reflecting the prevalent involvement of deep perforators. Before treatment with anti-TNF, thalidomide, or both, new ischemic lesions developed in half of patients at follow-up studies. ${ }^{4,7,13,15}$ Conversely, no new neurologic events or MR imaging lesions were noticed afterward, except for 2 recurrences shortly after introduction of anti-TNF or temporary discontinuation of thalidomide. Of note, the prevalence of ischemic strokes in our cohort was similar to that reported by Bulut et $\mathrm{al}^{14}$ in patients with DADA2-related vasculopathy and was expectedly higher than that described in a review also including patients with purely hematologic phenotypes (around 40\%). ${ }^{6}$ Interestingly, some patients presented with small lacunar strokes clearly depicted on DWI at clinical onset that were difficult to distinguish from enlarged perivascular spaces in follow-up examinations probably because of their small size and progressive gliotic "healing" changes. This suggests a possible underestimation of these subtle findings if brain MR imaging studies are not performed in the acute stage or with high spatial resolution sequences. As previously identified in a few patients with DADA2, ${ }^{14,15}$ we found an additional small ischemic medullary infarct at the cervical level in 1 patient. Of note, spinal cord involvement in individuals with DADA2 might also be underrated because only a minority of patients underwent a spinal MR imaging in both the current and in Bulut et al's series. ${ }^{14}$

Intracranial hemorrhagic strokes are additional complications of DADA2, ${ }^{1,2,6,11,12}$ involving nearly $12 \%$ of cases in a recent review $^{6}$ and $17 \%$ in our cohort. These hemorrhages may occur in isolation $^{1,12}$ or in association with ischemic strokes, ${ }^{2,12}$ as seen in our patients. In most published cases, no underlying causes were found. Conversely, 2 intracranial aneurysms were detected in 1 of our patients presenting with diffuse subarachnoid hemorrhage and a history of peripheral intracranial aneurysm and lobar hematoma. Of note, CNS aneurysms have been described only once in a patient with DADA2 ${ }^{1}$ and in a few patients with PAN who had unknown ADA2 status. ${ }^{16-18}$ These aneurysms are multiple, small $(<5 \mathrm{~mm})$, and peripheral, arising in nonbranching sites or rupturing even when small. ${ }^{16-18}$ Additionally, they can cause cranial nerve compression or infarction because of occlusion of the parental vessel. ${ }^{17}$ Their management is controversial, with both medical and surgical and interventional treatments proposed. ${ }^{17}$ In our case, early follow-up studies demonstrated spontaneous aneurysmal thrombosis with total exclusion from circulation and no new intracranial aneurysms after 5 years. Of note, no visceral aneurysms were found in this patient and in the other investigated patients.

In their series, Bulut et $\mathrm{al}^{14}$ described brain atrophy in more than $50 \%$ of cases, without further information regarding the temporal evolution of this imaging finding. Similarly, we found mild diffuse enlargement of CSF spaces, including the ventricular system, the cortical cerebral sulci, or both, in $42 \%$ of patients at first brain MR imaging. However, none of the patients with available follow-up studies presented signs of progressive atrophy.

The first novel finding in this series was the detection of small parenchymal foci of susceptibility effect in 2 patients with DADA2, likely corresponding to brain microhemorrhages. The latter have been shown to correlate with hemosiderin-laden perivascular macrophages and have been associated with progressive aging and multiple neurologic conditions, including small-vessel disease and CNS vasculitis. ${ }^{19}$ Another patient presented a similar focal susceptibility effect located in the interpeduncular fossa, likely in a vessel wall, that could have the same origin, considering the absence of intracranial aneurysms and subarachnoid hemorrhage.

Different from Bulut et al, ${ }^{14}$ we did not identify any parenchymal area of enhancement after gadolinium injection. However, in one-third of patients, we found another novel feature, which was the presence of irregular, contrast-enhancing tissue in the interpeduncular fossa, crural cistern, and inner auditory canal, surrounding the basilar, posterior cerebral arteries and AICA, 


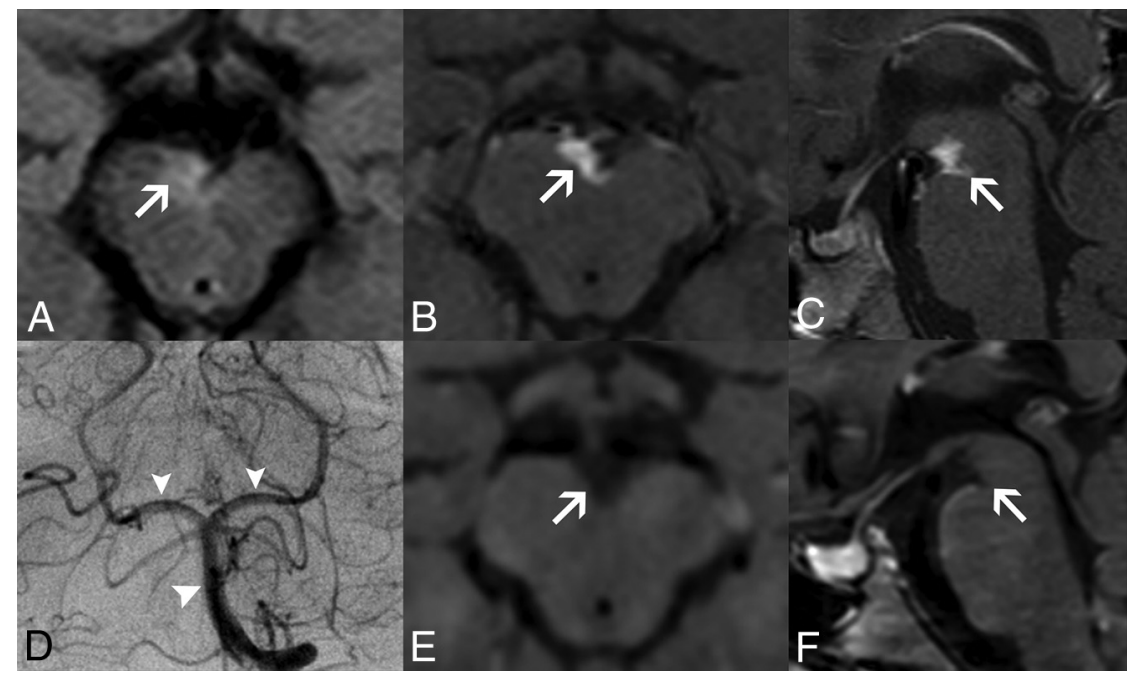

FIG 2. Perivascular enhancing tissue in adenosine deaminase 2 deficiency. Brain MR imaging in patient 1 at 8.5 years of age $(A-D)$ demonstrates a soft tissue component mass in the interpeduncular cistern surrounding the basilar artery and its terminal perforator branches with eccentric vessel wall involvement. The lesion is mildly hyperintense on axial FLAIR ( $A$, arrow), with intense solid enhancement on fat-saturated postgadolinium axial $(B)$ and sagittal $(C)$ TTWI sequences (arrows). DSA (D) reveals normal caliber of the basilar artery and posterior cerebral arteries (arrowheads). Postgadolinium axial $(E)$ and sagittal $(F)$ black-blood T1-weighted images performed at 9.7 years of age, after introduction of anti-TNF treatment, demonstrate a complete resolution of this likely inflammatory perivascular tissue (arrows).

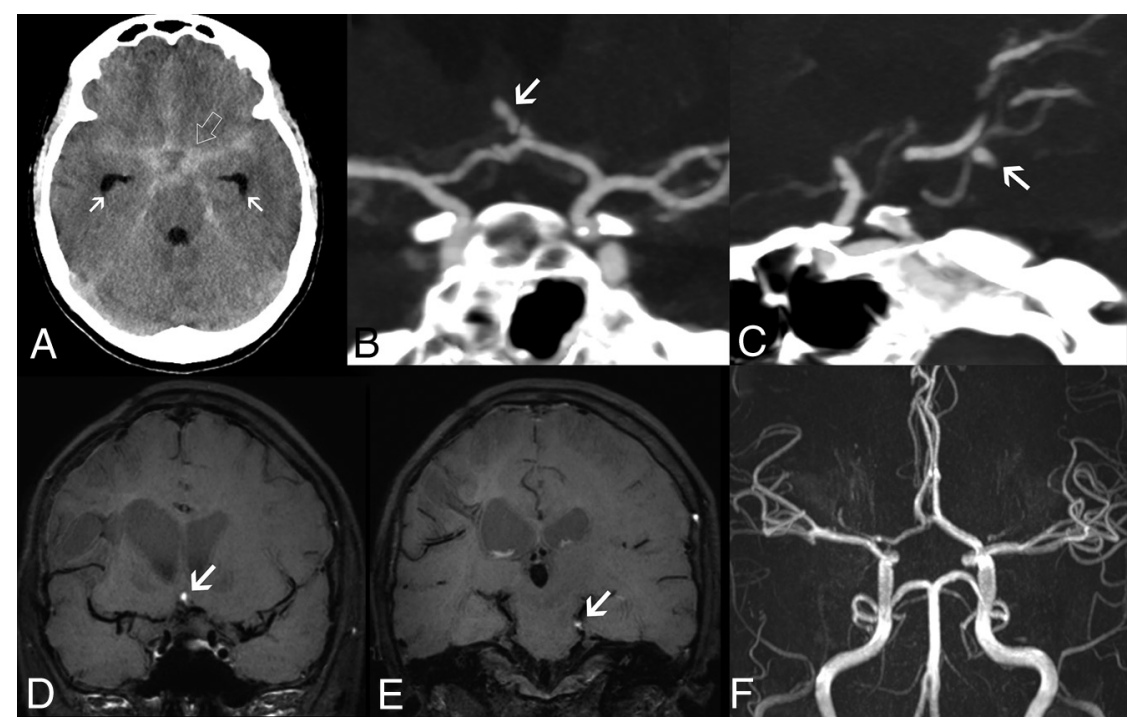

FIG 3. Intracranial aneurysms in adenosine deaminase deficiency. Axial unenhanced head CT scan in patient 5 at 16.4 years of age $(A)$ demonstrates a diffuse subarachnoid acute hemorrhage (SAH) (open arrow) symmetrically distributed in the basal cisterns, horizontal segments of the Sylvian fissures, and anterior interhemispheric fissure, with mild enlargement of the temporal horns in keeping with incipient hydrocephalus (arrows). CTA images $(B$ and $C$ ) reveal the presence of small-sized intracranial aneurysms of an anterior communicating artery branch (arrow) and the left superior cerebellar artery (arrow). Note that these 2 aneurysms have a peripheral location and arise in nonbranching sites. Follow-up brain MR imaging performed 23 days after the initial SAH episode, including coronal ( $D$ and E) black-blood TIWI, shows signs of intraaneurysmal thrombosis (arrows). TOF-MRA (F) does not depict flow-related signal within the aneurysmal sacs, in keeping with exclusion of the aneurysms from the circulation. These aneurysms remained excluded from circulation at 5-year follow-up. respectively. Eccentric vessel wall thickening and enhancement were noted in all patients, more pronounced at the origin of brain stem perforators, leading to progressive arterial stenosis in 1 patient. This pattern slightly differs from the concentric smooth arterial wall involvement typically seen in cerebral vasculitis caused by inflammation-related endothelial or vasa vasorum leakage. ${ }^{20}$ On the other hand, in 2 patients, this new finding was associated with acute ischemic infarcts in the territory of the posterior perforators, reflecting an active disease phase and suggesting an inflammatory origin. Accordingly, after anti-TNF treatment, there was total tissue regression in all patients and normalization in caliber of the stenosed artery at 1-year follow-up. As in the present series, most skin and bowel biopsy specimens of patients with DADA2 either reveal a PAN-like or a leukocytoclastic type of vasculitis. ${ }^{1,2,10}$ However, histologic proof of CNS vasculitis was not obtained so far. ${ }^{2}$ Therefore, the precise mechanism of DADA2related intracranial vasculopathy remains unknown and awaits further research. Interestingly, most of our patients presented unremarkable angiographic findings. Indeed, abnormalities of intracranial vessels have been only occasionally described in DADA2, ${ }^{14,21}$ probably caused by predominant involvement of mediumand small-sized vessels, frequently beyond the current resolution of lumen-based angiographic studies, including DSA. Accordingly, with the exception of the infarcted tissues, we did not find brain perfusion abnormalities on arterial spinlabeling.

Finally, we identified 2 DADA2related PRES events in our cohort. PRES has been occasionally reported in patients with DADA2, ${ }^{11,14}$ as well as in patients with PAN with unknown ADA2 status. ${ }^{22}$ Both our cases occurred during childhood and manifested with seizures associated with typical neuroimaging patterns. ${ }^{23}$ These events occurred in the context of arterial hypertension, a complication of DADA $2^{6}$ and a wellknown risk factor for $\mathrm{PRES}^{23}$; none of the patients was under anti-TNF treatment. Of note, ADA2 is an endothelial growth factor. ${ }^{2}$ Therefore, 
patients with DADA2 may be intrinsically more prone to endothelial dysfunction, which is the presumed key-role mechanism of PRES. ${ }^{23}$

\section{CONCLUSIONS}

We expanded the neuroimaging phenotype of DADA2, including (in addition to multiple, recurrent brain lacunar ischemic or hemorrhagic strokes, spinal infarcts, PRES events, and intracranial aneurysms) cerebral microbleeds, and a peculiar, likely inflammatory, perivascular tissue in the basal and prepontine cisterns. Together with early clinical onset, positive family history, inflammatory flares, and systemic abnormalities, these findings should raise the suspicion of DADA2, thus prompting genetic evaluation and institution of TNF inhibitors, with a potentially positive impact on neurologic outcome.

Disclosures: Ana Geraldo-UNRELATED: $12 \mathrm{M}$ research fellowship grant; UNRELATED: Payment for Manuscript Preparation: European Society of Neuroradiology, Comments: Money paid to individual author. Roberta CaorsiUNRELATED: Consultancy: Novartis, SOBI, Eli Lilly; Payment for Lectures Including Service on Speakers Bureaus: SOBI, Novartis. Antonella InsalacoUNRELATED: Board Membership: Novartis, SOBI, Comments: 1000 euros. Isabella Ceccherini-UNRELATED: Employment: Istituto Giannina Gaslini. Sara SignaRELATED: Grant: This work was supported by funds from Ricerca Corrente Disordini Neurologici e Muscolari (Linea 5) of the Italian Ministry of Health and by the Compagnia di San Paolo (Grant ROL 20573)*. Marco Gattorno-RELATED: Grant: Novartis, SOBI, Comments: Unrestricted grant to eurofever*; Consulting Fee or Honorarium: Novartis, SOBI; UNRELATED: Board Membership: Novartis, SOBI; Consultancy: Novartis, SOBI; Grants/Grants Pending: Novartis, SOBI, Comments: Unrestricted grant to eurofever*; Payment for lectures including service on speakers bureaus: Novartis, SOBl; Payment for Development of Educational Presentations: Novartis, SOBI. Mariasavina Severino-RELATED: Grant: Ricerca Corrente sui Disordini Neurologici e Muscolari (Linea 5) of the Italian Ministry of Health, Comments: This work was supported by funds from Ricerca Corrente sui Disordini Neurologici e Muscolari (Linea 5) of the Italian Ministry of Health*; UNRELATED: Employment: Neuroradiology consultant, IRCCS Istituto Giannina Gaslini. *Money paid to institution.

\section{REFERENCES}

1. Navon Elkan P, Pierce SB, Segel R, et al. Mutant adenosine deaminase 2 in a polyarteritis nodosa vasculopathy. $N$ Engl J Med 2014;370:921-31 CrossRef Medline

2. Zhou Q, Yang D, Ombrello AK, et al. Early-onset stroke and vasculopathy associated with mutations in ADA2. $N$ Engl $\mathrm{J} \mathrm{Med}$ 2014;370:911-20 CrossRef Medline

3. Bras J, Guerreiro R, Santo GC. Mutant ADA2 in vasculopathies. $N$ Engl J Med 2014;371:478-80 CrossRef Medline

4. Van Montfrans JM, Hartman EAR, Braun KPJ, et al. Phenotypic variability in patients with ADA2 deficiency due to identical homozygous R169Q mutations. Rheumatology (Oxford) 2016;55:902-10 CrossRef Medline

5. Lee PY, Kellner ES, Huang Y, et al. Genotype and functional correlates of disease phenotype in deficiency of adenosine deaminase 2 (DADA2). J Allergy Clin Immunol 2020;145:1664-72.e10 CrossRef Medline

6. Fayand A, Sarrabay G, Belot A, et al. Multiple facets of ADA2 deficiency: vasculitis, auto-inflammatory disease and im- munodeficiency: a literature review of 135 cases from literature. Rev Med Interne 2018;39:297-306 CrossRef Medline

7. Westendorp WF, Nederkoorn PJ, Aksentijevich I, et al. Unexplained early-onset lacunar stroke and inflammatory skin lesions: consider ADA2 deficiency. Neurology 2015;84:2092-93 CrossRef Medline

8. Nanthapisal S, Murphy C, Omoyinmi E, et al. Deficiency of adenosine deaminase type 2: a description of phenotype and genotype in fifteen cases. Arthritis Rheumatol 2016;68:2314-22 CrossRef Medline

9. Elbracht M, Mull M, Wagner N, et al. Stroke as initial manifestation of adenosine deaminase 2 deficiency. Neuropediatrics 2017;48:11114 CrossRef Medline

10. Caorsi R, Penco F, Grossi A, et al. ADA2 deficiency (DADA2) as an unrecognised cause of early onset polyarteritis nodosa and stroke: a multicentre national study. Ann Rheum Dis 2017;76:1648-56 CrossRef Medline

11. Özen S, Batu ED, Taş kiran EZ, et al. A monogenic disease with a variety of phenotypes: deficiency of adenosine deaminase 2. J Rheumatol 2020;47:117-25 CrossRef Medline

12. Sahin S, Adrovic A, Barut $\mathrm{K}$, et al. Clinical, imaging and genotypical features of three deceased and five surviving cases with ADA2 deficiency. Rheumatol Int 2018;38:129-36 CrossRef Medline

13. Caorsi R, Omenetti A, Morreale A, et al. Rapid and sustained effect of anti-TNF treatment in patients with ADA2 deficiency. Ped Rheumatol 2015;13:(Suppl) O80 CrossRef

14. Bulut E, Erden A, Karadag O, et al. Deficiency of adenosine deaminase 2; special focus on central nervous system imaging. $J$ Neuroradiol 2019;46:193-98 CrossRef Medline

15. Ganhão S, Loureiro GB, Oliveira DR, et al. Two cases of ADA2 deficiency presenting as childhood polyarteritis nodosa: novel ADA2 variant, atypical CNS manifestations, and literature review. Clin Rheumatol 2020;39:3853-60 CrossRef Medline

16. Toyoda K, Tsutsumi K, Hirao T, et al. Ruptured intracranial aneurysms in pediatric polyarteritis nodosa. Neurol Med Chir (Tokyo) 2012;52:928-932 CrossRef Medline

17. Boukobza M, Dossier A, Laissy JP. Thrombosed aneurysm of the posterior inferior cerebellar artery and lateral medullary ischemia as the initial presentation of polyarteritis nodosa: case report and literature review. J Stroke Cerebrovasc Dis 2018;27:e168-71 CrossRef Medline

18. Smith G, Hoh BL, Albayram MS. Anterior spinal artery aneurysm presenting with spinal subarachnoid hemorrhage in a case of polyarteritis nodosa. Clin Imaging 2019;56:108-13 CrossRef Medline

19. Wardlaw JM, Smith C, Dichgans M. Mechanisms of sporadic cerebral small vessel disease: insights from neuroimaging. Lancet Neurol 2013;12:483-97 CrossRef Medline

20. Mandell DM, Mossa-Basha M, Qiao Y, et al. Intracranial vessel wall MRI: principles and expert consensus recommendations of the American Society of Neuroradiology. AJNR Am J Neuroradiol 2017;38:218-29 CrossRef Medline

21. Garg N, Kasapcopur O, Foster J, et al. Novel adenosine deaminase 2 mutations in a child with a fatal vasculopathy. Eur J Pediatr 2014;173:827-30 CrossRef Medline

22. Navinan MR, Subasinghe CJ, Kandeepan T, et al. Polyarteritis nodosa complicated by posterior reversible encephalopathy syndrome: a case report. BMC Res Notes 2014;7:89 CrossRef Medline

23. Fugate JE, Rabinstein AA. Posterior reversible encephalopathy syndrome: clinical and radiological manifestations, pathophysiology, and outstanding questions. Lancet Neurol 2015;14:914-25 CrossRef Medline 